\title{
ON THE FIRST-ORDER EXPRESSIBILITY OF LATTICE PROPERTIES RELATED TO UNICOHERENCE IN CONTINUA
}

\author{
PAUL BANKSTON
}

\begin{abstract}
Many properties of compacta have "textbook" definitions which are phrased in lattice-theoretic terms that, ostensibly, apply only to the full closed-set lattice of a space. We provide a simple criterion for identifying such definitions that may be paraphrased in terms that apply to all lattice bases of the space, thereby making model-theoretic tools available to study the defined properties. In this note we are primarily interested in properties of continua related to unicoherence; i.e., properties that speak to the existence of "holes" in a continuum and in certain of its subcontinua.
\end{abstract}

\section{THE EXPRESSIBILITY LEMMA}

We continue our study [4] of compacta (i.e., compact Hausdorff spaces), especially of continua (i.e., connected compacta), from the perspective of model theory. Many of the classic definitions of properties pertaining to compacta are easily phrased in finitistic lattice-theoretic terms involving closed sets. (It is convenient to use closed - rather than, say, open - set phraseology because, in the setting of compacta, closed sets are compacta themselves.) For any space $X$, let $F(X)$ be the collection of all closed subsets of $X$, viewed as a bounded lattice; i.e., as a special structure for the first-order alphabet $L=\{\sqcup, \sqcap, \perp, \top\}$. As one would expect, $\sqcup$ is interpreted as union, $\sqcap$ as intersection, $\perp$ as $\emptyset$, and $\top$ as $X$. A sublattice $\mathcal{A}$ of $F(X)$ is a lattice base for $X$ if every member of $F(X)$ is an intersection of members of $\mathcal{A}$.

What makes the notion of lattice base so important in our study of compacta is the following Wallman-style representation theorem (see [11]): There is a sentence in the first-order language $\mathrm{L}_{\omega \omega}$ over $\mathrm{L}$, whose models are precisely the lattices that are isomorphic to the lattice bases for compacta.

The sentence alluded to above is quite easy to describe: In addition to stating the universal $\left(\Pi_{1}^{0}\right)$ condition of being a distributive lattice, it includes the two universalexistential $\left(\Pi_{2}^{0}\right)$ conditions of normality, $\forall x \forall y \exists x^{\prime} \exists y^{\prime}\left[(x \sqcap y=\perp) \rightarrow\left(\left(x \sqcap x^{\prime}=\right.\right.\right.$ $\left.\perp) \wedge\left(y \sqcap y^{\prime}=\perp\right) \wedge\left(x^{\prime} \sqcup y^{\prime}=\top\right)\right)$ ] (saying that two disjoint lattice elements may be separated by disjoint open sets whose complements are lattice elements), and disjunctivity, $\forall x \forall y \exists z[(x \neq y) \rightarrow(((z \neq \perp) \wedge(z \leq x) \wedge(z \sqcap y=\perp)) \vee((z \neq$ $\perp) \wedge(z \leq y) \wedge(z \sqcap x=\perp))$ )] (saying that for each two lattice elements, there is a non-bottom lattice element that is dominated by one and disjoint from the other). (We use the standard abbreviations " $x \neq y$ " for " $\neg(x=y)$ " and " $x \leq y$ " for " $x \sqcap y=x$ " (equivalently for " $x \sqcup y=y ")$.) If $\mathbf{A}$ is any L-structure that satisfies these conditions, and so is a normal disjunctive lattice, then $\mathbf{A}$ is naturally

2000 Mathematics Subject Classification. Primary 03C52, 54F15, 54F55; Secondary 03C20, 06D05, 54C10.

Key words and phrases. unicoherence, finite expressibility, lattice properties, continua. 
isomorphic to a lattice base for the compactum $S(\mathbf{A})$, defined by taking the maximal filters of $\mathbf{A}$ for points, and the sets $a^{\sharp}:=\{P \in S(\mathbf{A}): a \in P\}, a \in \mathbf{A}$, for basic closed sets. The space $S(\mathbf{A})$ is referred to as the maximal spectrum of $\mathbf{A}$, and is (up to homeomorphism) the only compactum for which $\mathbf{A}$ serves as a lattice base. The operation $S($ ) is contravariantly functorial, taking lattice homomorphisms to continuous maps (which are surjective when the homomorphisms are embeddings). And though $S($ ) is quite definitely "many-one" in general, it does indeed become Stone duality when restricted to the Boolean (i.e., complemented) lattices.

Let us now say that a sentence $\varphi$ in $\mathrm{L}_{\omega \omega}$ is an $F$-expression for a property/class $\mathfrak{P}$ of compacta if $\mathfrak{P}=\{X: F(X) \models \varphi\}$; a class is $F$-expressible if it has an $F$ expression. We call a sentence $\varphi$ base free if for any normal disjunctive lattice $\mathbf{A}$, $\mathbf{A} \models \varphi$ if and only if $F(S(\mathbf{A})) \models \varphi$ (i.e., if the truth of $\varphi$ in $\mathbf{A}$ depends only on the topological type of $\mathbf{A}$ ). If a class of compacta has a base free $F$-expression, we say the class is finitely expressible.

We call a sentence $\theta$ of $\mathrm{L}_{\omega \omega} F$-valid if $F(X) \models \theta$ for every compactum $X$. Then define a sentence $\varphi$ to be relatively base free if there is an $F$-valid sentence $\theta$ such that whenever $\mathbf{A}$ is a normal disjunctive lattice satisfying $\theta$, we have $\mathbf{A} \models \varphi$ if and only if $F(S(\mathbf{A})) \models \varphi$. (So relatively base free becomes base free when it is possible to choose $\theta$ to be valid for all normal disjunctive lattices.) When it becomes necessary to specify a sentence $\theta$ witnessing the relative base freeness of $\varphi$, we term $\theta$ a lever sentence for $\varphi$.

Our main model-theoretic "expressibility" lemma is largely a consequence of known results, principally the Frayne-Morel-Scott characterization of elementary classes (see, e.g., [6]).

Lemma 1.1. A property of compacta is finitely expressible if (and only if) it has a relatively base free $F$-expression.

Proof. A property $\mathfrak{P}$ of compacta is finitely expressible just in case the class $S^{-1}[\mathfrak{P}]:=\{\mathbf{A}: \mathbf{A}$ is a normal disjunctive lattice and $S(\mathbf{A}) \in \mathfrak{P}\}$ is the class of models of a single sentence of $\mathrm{L}_{\omega \omega}$. And this condition is true just in case a certain classic ultraproduct criterion is satisfied (see [6]), namely that, for any indexed family $\left\{\mathbf{A}_{i}: i \in I\right\}$ and any ultrafilter $\mathcal{D}$ on $I$, the ultraproduct $\prod_{\mathcal{D}} \mathbf{A}_{i}$ is in $S^{-1}[\mathfrak{P}]$ if and only if $\left\{i \in I: \mathbf{A}_{i} \in S^{-1}[\mathfrak{P}]\right\} \in \mathcal{D}$.

So let $\varphi$ be a relatively base free $F$-expression for the property $\mathfrak{P}$ of compacta, and suppose $\theta$ is a lever sentence for $\varphi$. Let $\mathcal{D}$ be an ultrafilter on the index set $I$, and $\left\{\mathbf{A}_{i}: i \in I\right\}$ an $I$-indexed collection of L-structures such that $J=\{i \in I$ : $\left.\mathbf{A}_{i} \in S^{-1}[\mathfrak{P}]\right\} \in \mathcal{D}$. Then for each $i \in J, F\left(S\left(\mathbf{A}_{i}\right)\right) \models \varphi$; hence $\prod_{\mathcal{D}} F\left(S\left(\mathbf{A}_{i}\right)\right) \models \varphi$ as well. But $\theta$ is $F$-valid, so $\prod_{\mathcal{D}} F\left(S\left(\mathbf{A}_{i}\right)\right) \models \theta$. Thus $F\left(S\left(\prod_{\mathcal{D}} F\left(S\left(\mathbf{A}_{i}\right)\right)\right) \models \varphi\right.$ because $\theta$ is a lever sentence for $\varphi$.

Now the space $S\left(\prod_{\mathcal{D}} \mathbf{A}_{i}\right)$, also denoted $\sum_{\mathcal{D}} S\left(\mathbf{A}_{i}\right)$, is the topological ultracoproduct introduced in [1]. The notation is justified because the ultracoproduct construction depends only on the constituent compacta, not on the particular choice of lattice bases for them. In particular, the spaces $S\left(\prod_{\mathcal{D}} \mathbf{A}_{i}\right)$ and $S\left(\prod_{\mathcal{D}} F\left(S\left(\mathbf{A}_{i}\right)\right)\right)$ are homeomorphic; hence we infer that $F\left(S\left(\prod_{\mathcal{D}} \mathbf{A}_{i}\right)\right) \models \varphi$. Thus $\prod_{\mathcal{D}} \mathbf{A}_{i} \in S^{-1}[\mathfrak{P}]$, as desired.

If $J \notin \mathcal{D}$, then $I \backslash J \in \mathcal{D}$. For each $i \in I \backslash J, F\left(S\left(\mathbf{A}_{i}\right)\right) \models \neg \varphi$. Thus $\prod_{\mathcal{D}} F\left(S\left(\mathbf{A}_{i}\right)\right) \models \neg \varphi$. As above, we have $\prod_{\mathcal{D}} F\left(S\left(\mathbf{A}_{i}\right)\right) \models \theta$, and hence 
$F\left(S\left(\prod_{\mathcal{D}} F\left(S\left(\mathbf{A}_{i}\right)\right)\right)\right) \models \neg \varphi$. Thus $F\left(S\left(\prod_{\mathcal{D}} \mathbf{A}_{i}\right)\right) \models \neg \varphi$; so we have $\prod_{\mathcal{D}} \mathbf{A}_{i} \notin$ $S^{-1}[\mathfrak{P}]$.

Remark 1.2. As the terminology ultracoproduct suggests, $\sum_{\mathcal{D}} X_{i}$ is the limit of the inverse system $\left\{\beta\left(\bigcup_{i \in J}\left(X_{i} \times\{i\}\right)\right): J \in \mathcal{D}\right\}$ of coproducts in the category of compacta and continuous maps. ( $\mathcal{D}$ is ordered by reverse inclusion; the bonding maps are Stone-Čech lifts of the obvious inclusion maps.) If $q^{\beta}$ denotes the StoneCech lift of the projection map from $\bigcup_{i \in I}\left(X_{i} \times\{i\}\right)$ to the discrete space $I$, then $\sum_{\mathcal{D}} X_{i}$ may be viewed as the $q^{\beta}$-fiber over $\mathcal{D} \in \beta(I)$. Furthermore, if each $X_{i}$ is the same space $X$, then there is also the projection map $p: X \times I \rightarrow X$. The restriction $p_{X, \mathcal{D}}$ of $p^{\beta}$ to the ultracopower $X_{\mathcal{D}}=\sum_{\mathcal{D}} X_{i} \subseteq \beta(X \times I)$ is the canonical codiagonal map, a continuous surjection onto $X=\beta(X)$. The codiagonal map is dual to the usual diagonal embedding from a relational structure into an ultrapower of that structure, and may be used to define classes of continuous surjections that mirror well-known classes of embeddings in model theory. Of particular interest is the class of co-existential maps, first introduced in [2], and mirroring the existential embeddings. These are maps $f: X \rightarrow Y$ between compacta such that there is an ultrafilter $\mathcal{D}$ on a set $I$ and a continuous surjection $g: Y_{\mathcal{D}} \rightarrow X$ such that $f \circ g=p_{Y, \mathcal{D}}$. We will have more to say about co-existential maps in Section 5 .

\section{A USEFUL LEVER SENTENCE}

In this section we describe a sentence $\theta$ that serves as a lever sentence for a number of familiar $F$-expressions. Roughly speaking, $\theta$ says of a lattice base that it is atomic, and that it contains enough components. To make this precise, we first state some well-known facts about subcontinua of compacta; then introduce three very useful formulas in $L_{\omega \omega}$.

(C1) Let $\mathcal{A}$ be a lattice base for $X$, with $A \in \mathcal{A}$. If $R$ and $S$ are disjoint closed sets such that $A=R \cup S$, then both $R$ and $S$ are in $\mathcal{A}$. In particular, a clopen subset of $X$ is in every lattice base for $X$.

(C2) If $a, b \in X$ lie in different components of $X$, then there is a clopen set $A$ of $X$ such that $a \in A$ and $b \notin A$.

From $\mathrm{C} 2$ above, we may proceed to the following two facts:

(C3) If $A$ and $B$ are closed subsets of $X$, then there is a clopen set containing one and disjoint from the other if and only if there is no connected subset of $X$ that intersects both $A$ and $B$.

(C4) If $A$ and $B$ are disjoint closed subsets of $X$ and one of the two is a component of $X$, then there is a clopen set containing one and disjoint from the other.

Now for the formulas we wish to use. 
(F1) $\alpha(x)$ says of an element that it is an atom; i.e., it is the $\Pi_{1}^{0}$ formula

$$
\forall y[((x \neq \perp) \wedge(y \leq x) \wedge(y \neq \perp)) \rightarrow(y=x)]
$$

If $\mathcal{A}$ is a lattice base for a compactum $X$ and $A \in \mathcal{A}$, then we write $\alpha[A]$ to denote the sentence obtained by substituting each occurrence of the variable $x$ in $\alpha(x)$ by a new constant representing $A$. Then it is a triviality to show that $\mathcal{A} \models \alpha[A]$ if and only if $A$ is a singleton subset of $X$.

(F2) $\gamma(x)$ says of an element that it is connected; i.e., it is the $\Pi_{1}^{0}$ formula

$$
\forall y \forall z[((x=y \sqcup z) \wedge(y \sqcap z=\perp)) \rightarrow((y=\perp) \vee(z=\perp))]
$$

If $\mathcal{A}$ is a lattice base for a compactum $X$ and $A \in \mathcal{A}$, then $\mathcal{A} \models \gamma[A]$ if and only if $A$ is a connected subset of $X$. [This follows from $\mathrm{C} 1$ above.]

(F3) $\kappa(z, x)$ says of two elements that the first is a component of the second; i.e., it is the $\Pi_{2}^{0}$ formula

$$
\begin{gathered}
\forall w \exists r \exists s[(z \leq x) \wedge \gamma(z) \wedge[((w \leq x) \wedge(w \sqcap z=\perp)) \rightarrow \\
((w \leq r) \wedge(z \leq s) \wedge(r \sqcup s=x) \wedge(r \sqcap s=\perp))]]
\end{gathered}
$$

If $\mathcal{A}$ is a lattice base for a compactum $X$ and $A, C \in \mathcal{A}$, then $\mathcal{A} \models \kappa[C, A]$ if and only if $C$ is a component of $A$. [This follows from $\mathrm{C} 3$ and $\mathrm{C} 4$ above.]

At this point we let $\theta$ be the $\Pi_{4}^{0}$ sentence $\alpha \wedge \kappa$, where: $\alpha$ is the sentence $\forall x \exists y[(x \neq$ $\perp) \rightarrow((y \leq x) \wedge \alpha(y))]$, saying a lattice is atomic; and $\kappa$ is the sentence $\forall x \forall y \exists z[((y \leq$ $x) \wedge \alpha(y)) \rightarrow((y \leq z) \wedge \kappa(z, x))]$, saying that when a lattice element dominates an atomic element, the component of the former containing the latter is also a lattice element.

We sum up the discussion in this section with the following.

Lemma 2.1. Let $\theta$ be the sentence $\alpha \wedge \kappa$ described above. Then $\theta$ is F-valid. Furthermore a lattice base $\mathcal{A}$ for a compactum $X$ satisfies $\theta$ if and only if: (i) whenever $A \in \mathcal{A}$ is nonempty, there is some $a \in A$ such that $\{a\} \in \mathcal{A}$; and (ii) whenever $A \in \mathcal{A}$ and $a \in A$ is such that $\{a\} \in \mathcal{A}$, it is the case that the component of $A$ containing a is an element of $\mathcal{A}$.

\section{FINITELY EXPRESSING UNICOHERENCE}

In [4] we exhibited base free $\Pi_{2}^{0} F$-expressions for various well-known properties of compacta. These include: (i) having covering dimension $\leq n, n=0,1, \ldots$ (Proposition 3.2); (ii) being an indecomposable continuum (Corollary 4.6); and (iii) being a hereditarily indecomposable continuum (Corollary 4.10). As a result of techniques 
developed in [4] (Theorem 1.2, inspired by the Chang-Łoś-Suszko theorem), these properties are then automatically closed under co-existential images, as well as the taking of limits of inverse systems with continuous surjections for bonding maps.

By using a rather cumbersome ultracoproduct argument (Theorem 5.1 in [4]), we were able to give a nonconstructive proof that the properties of being a continuum of multicoherence degree $\leq n, n=0,1, \ldots$ are also finitely expressible. This result, together with S. B. Nadler's theorem [10] that multicoherence degree cannot be raised by the taking of inverse limits with continuous surjections for bonding maps, shows that, for $n=0,1, \ldots$, there is a base free $\Pi_{2}^{0}$ sentence $\mu_{n}$ that finitely expresses multicoherence degree $\leq n$. It is still an open problem, however, to formulate these sentences $\mu_{n}$ explicitly. Such formulations are desirable because of their strong potential for providing new and informative characterizations of properties.

In this section we illustrate how the expressibility lemma (1.1) may be used to provide a more streamlined (but still nonconstructive) proof of the existence of base free $F$-expressions for the class of continua of multicoherence degree $\leq n$, for any fixed natural number $n$. For simplicity we focus on the case $n=0$, there being no essential loss of generality in doing so. This gives us the class of continua that are unicoherent; i.e., those continua $X$ satisfying the condition that if $X=K \cup M$, where $K$ and $M$ are subcontinua, then $K \cap M$ is connected. Cells, dendrites and indecomposable continua are unicoherent; simple closed curves and figure-eights are not. (Indeed, they have multicoherence degree 1 and 2, respectively.)

Before proceeding, we record the following useful technical result.

Lemma 3.1. Let $X$ be a compactum, with $K, M, R$ and $S$ closed subsets satisfying: (i) $R$ and $S$ are nonempty and disjoint; and (ii) $K \cap M=R \cup S$ (so $\{R, S\}$ is a disconnection of $K \cap M)$. Let $\mathcal{A}$ be a lattice base for $X$. Then there are $K^{*}$, $M^{*}, R^{*}$ and $S^{*}$, all members of $\mathcal{A}$, such that: (a) $K \subseteq K^{*}, M \subseteq M^{*}, R \subseteq R^{*}$ and $S \subseteq S^{*}$; and (b) $\left\{R^{*}, S^{*}\right\}$ is a disconnection of $K^{*} \cap M^{*}$.

Proof. We first find open sets $U$ and $V$ such that $R \subseteq U, S \subseteq V$, and the closures $\bar{U}$ and $\bar{V}$ are disjoint. Set $K^{\prime}=K \backslash(U \cup V)$ and $M^{\prime}=M \backslash(U \cup V)$. Then $K^{\prime}$ and $M^{\prime}$ are disjoint closed sets, so find $K^{+}, M^{+} \in \mathcal{A}$ such that $K^{\prime} \subseteq K^{+}, M^{\prime} \subseteq M^{+}$ and $K^{+} \cap M^{+}=\emptyset$. Next we find $R^{*}, S^{*} \in \mathcal{A}$ such that $\bar{U} \subseteq R^{*}, \bar{V} \subseteq S^{*}$, and $R^{*} \cap S^{*}=\emptyset$. Finally we set $K^{*}=K^{+} \cup\left(R^{*} \cup S^{*}\right)$ and $M^{*}=M^{+} \cup\left(R^{*} \cup S^{*}\right)$, both sets in $\mathcal{A}$.

We have immediately that $R \subseteq U \subseteq \bar{U} \subseteq R^{*}$; similarly $S \subseteq S^{*}$. Also $K \subseteq$ $K^{\prime} \cup(U \cup V) \subseteq K^{+} \cup(U \cup V) \subseteq K^{+} \cup\left(R^{*} \cup S^{*}\right)=K^{*}$; similarly $M \subseteq M^{*}$.

Finally $K^{*} \cap M^{*}=\left(K^{+} \cap M^{+}\right) \cup\left(R^{*} \cup S^{*}\right)=R^{*} \cup S^{*}$, so $\left\{R^{*}, S^{*}\right\}$ is a disconnection of $K^{*} \cap M^{*}$.

Let us now formulate the textbook definition of unicoherence as the $\Pi_{2}^{0} F$-expression $v$ :

$$
\forall x \forall y[\gamma(\top) \wedge((\gamma(x) \wedge \gamma(y) \wedge(x \sqcup y=\top)) \rightarrow \gamma(x \sqcap y))]
$$


PAUL BANKSTON

The following theorem is a paraphrase of earlier work [4], but the proof is new and in a form that allows for easy generalization.

Theorem 3.2. The F-expression $v$ given above is relatively base free; hence the property of unicoherence in continua is finitely expressible.

Proof. We recall the $F$-valid sentence $\theta$ of Lemma 2.1 and show it is a lever sentence for $v$.

Let $X$ be a continuum, with $\mathcal{A}$ a lattice base for $X$ that is a model of $\theta$. Clearly if $\mathcal{A} \models \neg v$, then $F(X) \models \neg v$ too (whether or not $\mathcal{A} \models \theta$ ). For the converse, suppose that $F(X) \models \neg v$. Then there are subcontinua $K$ and $M$ of $X$ such that $K \cup M=X$ and $K \cap M=R \cup S$, where $R$ and $S$ are disjoint nonempty closed sets. By Lemma 3.1, there are $K^{*}, M^{*}, R^{*}, S^{*}$ in $\mathcal{A}$ such that $K \subseteq K^{*}, S \subseteq S^{*}, R \subseteq R^{*}, S \subseteq S^{*}$, and $\left\{R^{*}, S^{*}\right\}$ is a disconnection of $K^{*} \cap M^{*}$.

Since both $K$ and $M$ are connected, $X=K \cup M$, and $K \cap M$ is disconnected, we know that both $K \backslash M$ and $M \backslash K$ are nonempty open subsets of $X$. In particular, both $K$ and $M$ have nonempty interiors in $X$. Since $\mathcal{A}$ is a lattice base, there exist nonempty $A$ and $B$ in $\mathcal{A}$ such that $A \subseteq K$ and $B \subseteq M$. And since $\mathcal{A}$ is atomic, there exist $a \in A \subseteq K$ and $b \in B \subseteq M$ such that both $\{a\}$ and $\{b\}$ are members of $\mathcal{A}$. Since $\mathcal{A}$ also satisfies the component condition given in Lemma 2.1 (ii), we know that the component $C$ of $K^{*}$ containing $a$ and the component $D$ of $M^{*}$ containing $b$ are both elements of $\mathcal{A}$. Since $K$ is a connected subset of $K^{*}$ containing $a$, we know $K \subseteq C$; likewise we know $M \subseteq D$. Thus $C \cup D=X$ and $R \cup S \subseteq C \cap D \subseteq R^{*} \cup S^{*}$. Since $R \subseteq R^{*}$ and $S \subseteq S^{*}$, we infer that $\left\{R^{*} \cap(C \cap D), S^{*} \cap(C \cap D)\right\}$ is a disconnection of $C \cap D$. Thus $\mathcal{A} \models \neg v$.

\section{FINITELY EXPRESSING STRONGER KINDS OF UNICOHERENCE}

A continuum is hereditarily unicoherent if every subcontinuum is unicoherent. This is equivalent to saying that the intersection of any two subcontinua is connected; and we can phrase this with the following $\Pi_{2}^{0} F$-expression, which we denote $\chi$ :

$$
\forall x \forall y[\gamma(\top) \wedge((\gamma(x) \wedge \gamma(y)) \rightarrow \gamma(x \sqcap y))]
$$

It is an open question whether hereditary unicoherence is finitely expressible; there are, however, two reasonably nontrivial reasons to believe in a positive answer. Firstly, hereditary unicoherence behaves in some important ways like the known finitely expressible property of hereditary indecomposability (specified by saying that the intersection of any two overlapping subcontinua is one or the other): both properties are closed under co-existential images, both are closed under the taking of inverse limits with surjective bonding maps. (See discussions in [4] and [5].) In particular, hereditary unicoherence is finitely expressible using a $\Pi_{2}^{0}$ sentence, if it is finitely expressible at all. The second reason is the half-century-old re-

sult of L. Gillman and M. Henriksen ([8]), saying that the Stone-Cech remainder 
$[0,1)^{*}:=\beta[0,1) \backslash[0,1)$ of the half-open unit interval is hereditarily unicoherent. It follows that when the index set is countable, an ultracopower of the closed unit interval - easily seen to be embeddable in $[0,1)^{*}$ — is hereditarily unicoherent as well.

Remark 4.1. Let us call two compacta elementarily tolerant if some lattice base for one is elementarily equivalent to some lattice base for the other. (This relation is reflexive and symmetric, as befits the accepted definitaion of tolerance relations. It may or may not be transitive, but its two-fold composition is an equivalence relation.) Using Löwenheim-Skolem arguments (see, e.g., [4]), it is possible to construct a metrizable continuum $X$, elementarily tolerant to $[0,1]$, such that $X$ fails to be either locally connected or hereditarily decomposable. This $X$ may be realized as a co-existential image of an ultracopower of $[0,1]$ via an ultrafilter on a countable index set, and so it is also hereditarily unicoherent because of the Gillman-Henriksen theorem cited above.

A continuum is weakly hereditarily unicoherent if the intersection of any two subcontinua possessing nonempty interiors is connected. This property is clearly weaker than hereditary unicoherence in general, but the two properties actually agree on the class of arcwise connected continua (see [7]). It is easy to modify the sentence $\chi$ above and thus give a $\Pi_{2}^{0} F$-expression for this property, which we denote $\chi^{w}$ :

$$
\begin{gathered}
\forall x \forall y \forall u \forall v[\gamma(\top) \wedge((\gamma(x) \wedge \gamma(y) \wedge((u \neq \top) \wedge \\
(v \neq \top) \wedge(u \sqcup x=\top) \wedge(v \sqcup y=\top))) \rightarrow \gamma(x \sqcap y))]
\end{gathered}
$$

With trivial modifications of the proof of Theorem 3.2, we have the following.

Theorem 4.2. The F-expression $\chi^{w}$ given above is relatively base free; hence the property of weak hereditary unicoherence in continua is finitely expressible.

Proof. In the proof of 3.2, we use the fact that $X=K \cup M$ only to infer that both $K$ and $M$ have nonempty interiors. This condition is given to us here.

Remark 4.3. The question arises as to the possible level of complexity of a base free $F$-expression that could be used to replace $\chi^{w}$. We show in the next section that a $\Pi_{2}^{0}$ sentence is possible.

A continuum is strongly unicoherent if it is unicoherent, and whenever it is the union of two proper subcontinua, each of those subcontinua is unicoherent also. In [9] T. Maćkowiak proves this definition equivalent to saying that any subcontinuum with nonempty interior is unicoherent. This shows clearly that strong unicoherence lies between hereditary unicoherence and weak hereditary unicoherence. (Thus all three properties coincide in the presence of arcwise connectedness. Also strong unicoherence and weak hereditary unicoherence coincide in the presence of hereditary decomposability (see [7]).) 
The reader will have no difficulty formulating $F$-expressions for either characterization above. However, attempts to extend the methods of Theorem 3.2 to show the finite expressibility of strong unicoherence seem to break down much as they do with hereditary unicoherence. In the next section we put some positive spin on the issue by showing all four unicoherence properties to be closed under images of co-existential maps and limits of inverse systems with surjective bonding maps.

\section{UNICOHERENCE PROPERTIES AND CO-EXISTENTIAL MAPS}

We recall the following basic result about co-existential maps (see Theorem 5.15 in $[3])$.

Theorem 5.1. Let $f: X \rightarrow Y$ be a co-existential map between compacta. Then there is a U-semilattice homomorphism $f^{*}: F(Y) \rightarrow F(X)$ such that:

(1) $f^{*}(K)$ is connected (resp., a singleton) whenever $K \in F(X)$ is connected (resp., a singleton).

(2) $f^{-1}[U] \subseteq f^{*}(K)$ whenever $K \in F(X)$ and $U \subseteq K$ is open in $Y$.

(3) $f \mid f^{*}(K)$ is a co-existential map from $f^{*}(K)$ onto $K$ for each $K \in F(Y)$.

Let us call the function $f^{*}$ (which depends on the ultracopower of $Y$ that witnesses the co-existentiality of $f$ ) a subinverse for $f$. Subinverses preserve finite unions, but do not seem to preserve finite intersections, and hence apparently fall short of being lattice homomorphisms. They do come close, though, in the following sense.

Corollary 5.2. Let $f: X \rightarrow Y$ be a co-existential map between compacta, with $f^{*}$ a subinverse for $f$. If $K$ and $M$ are subcompacta of $Y$, then $f\left[f^{*}(K) \cap f^{*}(M)\right]=$ $K \cap M$.

Proof. By virtue of the fact that $f$ is a function, we have $f\left[f^{*}(K) \cap f^{*}(M)\right] \subseteq$ $f\left[f^{*}(K)\right] \cap f\left[f^{*}(M)\right]$. The right-hand side is just $K \cap M$, by Theorem 5.1 (3). On the other hand, by the fact that $f^{*}$ is $\cup$-preserving, it respects inclusion. Hence $f\left[f^{*}(K) \cap f\left[f^{*}(M)\right] \supseteq f\left[f^{*}(K \cap M)\right]=K \cap M\right.$, again by 5.1 (3). This gives us the desired equality.

In [4] we used the finite expressibility of multicoherence degree $\leq n$ in continua, plus Nadler's theorem (Theorem 1 in [10]), to the effect that multicoherence degree is not raised by the taking of inverse limits with surjective bonding maps, to infer that co-existential maps do not raise multicoherence degree. We can prove this directly - and hence give an alternative (though round-about) proof of Nadler's preservation result - with the aid of Corollary 5.2.

Theorem 5.3. Let $\mathfrak{P}$ be any one of the properties: unicoherence, hereditary unicoherence, weak hereditary unicoherence, strong unicoherence. If $X$ is a continuum satisfying property $\mathfrak{P}$, and if $f: X \rightarrow Y$ is a co-existential map, then $Y$ satisfies $\mathfrak{P}$ as well. 
Proof. We prove the easy case where $\mathfrak{P}$ is weak hereditary unicoherence; the arguments for the other cases are similar, but even easier. Suppose $K$ and $M$ are subcontinua of $Y$, each with nonempty interior, and let $f^{*}: F(Y) \rightarrow F(X)$ be a subinverse for $f$. By Theorem 5.1, both $f^{*}(K)$ and $f^{*}(M)$ are subcontinua of $X$, each with nonempty interior. Since $X$ is weakly hereditarily unicoherent, $f^{*}(K) \cap f^{*}(M)$ is connected. By Corollary 5.2, so is $K \cap M$.

Remarks 5.4. (1) Observe that Theorem 5.3 may be generalized to the preservation of multicoherence degree $\leq n$ by noting that the number of components in the image of a continuous map cannot exceed the number of components in the domain.

(2) If we are given an inverse system of continua, each with property $\mathfrak{P}$, and where the bonding maps are continuous surjections, then we may infer that the inverse limit has property $\mathfrak{P}$, as long as $\mathfrak{P}$ is finitely expressible and is preserved under co-existential images. This works when $\mathfrak{P}$ is either unicoherence (already proved in [10]) or weak hereditary unicoherence (possibly new), but not in the other cases listed in Theorem 5.3. However, it is still true that these properties are preserved under the appropriate inverse limits: In the case of hereditary unicoherence, there is a straightforward proof in [10]; in the case of strong unicoherence, one uses the defining condition given above, plus the argument in [10].

(3) It is easy to see that Corollary 5.2 holds for arbitrary intersections, not just finite ones-if $\left\{K_{i}: i \in I\right\}$ is any family of subcompacta of $Y$, then $f\left[\bigcap_{i \in I} f^{*}\left(K_{i}\right)\right]=\bigcap_{i \in I} K_{i}$ - and is a trivial consequence of the questionable assertion that $\bigcap_{i \in I} f^{*}\left(K_{i}\right)=f^{*}\left(\bigcap_{i \in I} K_{i}\right)$. We do not know whether subinverses of co-existential maps preserve finite intersections, but they do not preserve infinite ones. Indeed, suppose $f: X \rightarrow Y$ is a coexistential map between compacta, with $y \in Y$ and $\left\{K_{i}: i \in I\right\}$ the family of all closures of open neighborhoods of $y$. Then, by Theorem 5.1 (2), $\bigcap_{i \in I} f^{*}\left(K_{i}\right) \supseteq f^{-1}[\{y\}]$ (actually, equality holds); while $\bigcap_{i \in I} K_{i}=\{y\}$. Thus, by Theorem $5.1(1), f^{*}\left(\bigcap_{i \in I} K_{i}\right)=f^{*}(\{y\})$ is a singleton; so the only way for $f^{*}$ to preserve arbitrary intersections is for $f$ to be a homeomorphism.

\section{REFERENCES}

[1] P. Bankston, Reduced coproducts of compact Hausdorff spaces, J. Symbolic Logic 52 (1987), 404-424.

[2] _ A hierarchy of maps between compacta, J. Symbolic Logic, 64 (1999), 1628-1644.

[3] _ A survey of ultraproduct constructions in general topology, Topology Atlas Invited Contributions 8 (2003), 1-32.

[4] - The Chang-Eoś-Suszko theorem in a topological setting, Archive for Mathematical Logic, 45 (2006), 97-112.

[5] _ Dendrites, topological graphs, and 2-dominance Houston J. Math., 35 (2009), no. 4, $1091-1102$.

[6] C. C. Chang and H. J. Keisler, Model Theory, third ed., North Holland, Amsterdam, 1990.

[7] J.J. Charatonik, Unicoherence and multicoherence, in: Encyclopedia of General Topology (K. P. Hart, J. Nagata, J. E. Vaughan, eds.), Elsevier, 2004, pp. 331-333. 
[8] L. Gillman and M. Henriksen, Rings of continuous functions in which every finitely generated ideal is principal, Trans. Amer. Math. Soc. 82 (1956), 366-391.

[9] T. Maćkowiak, Some kinds of the unicoherence, Comment. Math. Prace Mat. 20 (1977/78), 405-408.

[10] S. B. Nadler, Jr., Multicoherence techniques applied to inverse limits, Trans. Amer. Math. Soc. 157 (1971), 227-234.

[11] H. Wallman, Lattices and topological spaces, Ann. Math. (2) 39 (1938), 112-126.

Department of Mathematics, Statistics and Computer Science, Marquette UniverSity, Milwaukee, WI 53201-1881, U.S.A.

E-mail address: paulb@mscs.mu.edu 